DETERMINACIÓN DE LA DOSIS LETAL MEDIA DE LOS

Artículo Original

EXTRACTOS DE Paullinia clavigera var bullata Simpson

y Solanum mammosum L. PARA CONTROLAR ÁFIDOS EN CONDICIONES DE LABORATORIO

\title{
DETERMINATION OF THE LETAL AVERAGE DOSE OF THE EXTRACTS OF Paullinia clavigera var bullata Simpson and Solanum mammosum L. TO CONTROL AIDES IN LABORATORY
}

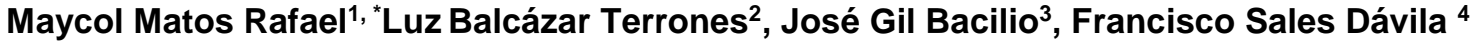

1 Universidad Nacional Agraria de la Selva. (UNAS), Huánuco, Tingo María - Perú.

2 Dirección de Investigación de Diversidad Biológica Terrestre Amazónica. Instituto de Investigaciones de la Amazonia Peruana / Facultad de Agronomía. Universidad Nacional Agraria de la Selva. (UNAS), Huánuco, Tingo María - Perú.

${ }^{3}$ Facultad de Agronomía. Universidad Nacional Agraria de la Selva. (UNAS), Huánuco, Tingo María - Perú.

${ }^{4}$ Instituto de Investigaciones de la Amazonia Peruana IIAP - Huánuco, Tingo María - Perú.

*Autor para correspondencia: Ibalcazar@iiap.gob.pe

Recibido: 22 de Octubre, 2019. Aceptado: 17 de Diciembre, 2019.

\section{RESUMEN}

Para determinar la dosis letal media $\left(\mathrm{DL}_{50}\right)$ en condiciones de laboratorio de los extractos de Paullinia clavigera y Solanum mammosum en la mortalidad de áfidos, se aplicaron los tratamientos: $\mathrm{T}_{1}\left(\mathrm{a}_{1} \mathrm{~b}_{1}=S\right.$. mammosum 25 $\%), T_{2}\left(a_{1} b_{2}=S\right.$. mammosum $\left.50 \%\right), T_{3}\left(a_{1} b_{3}=S\right.$. mammosum $\left.75 \%\right), T_{4}\left(a_{1} b_{4}=S\right.$. mammosum $\left.100 \%\right), T_{5}\left(a_{2} b_{1}=P\right.$. clavigera $25 \%), \mathrm{T}_{6}\left(\mathrm{a}_{2} \mathrm{~b}_{2}=\right.$ P. clavigera $\left.50 \%\right), \mathrm{T}_{7}\left(\mathrm{a}_{2} \mathrm{~b}_{3}=\right.$ P. clavigera $\left.75 \%\right), \mathrm{T}_{8}\left(\mathrm{a}_{2} \mathrm{~b}_{4}=\right.$ P. clavigera $\left.100 \%\right)$ y $\mathrm{T}_{9}$ (testigo); se utilizó el diseño completo al azar con arreglo factorial, de $2 \times 4+1$ testigo adicional, con cinco repeticiones. Para la dosis letal $\mathrm{DL}_{50}$, los resultados de mortalidad se ajustaron a los modelos probit y cúbico. Se evaluó: mortalidad acumulada, número de población de áfidos vivos por tratamiento y tiempo de la aplicación biocida a la muerte de los áfidos. El factor dosis influenció significativamente en la mortalidad de áfidos en comparación con el factor biocida que resultó no significativo; cuando interactuaron los factores las respuestas a la mortalidad fueron diferentes, los tratamientos $\mathrm{a}_{1} \mathrm{~b}_{4}$ (S. mammosum $\left.100 \%\right), \mathrm{a}_{2} \mathrm{~b}_{4}$ (S. clavigera $\left.100 \%\right)$ y $\mathrm{a}_{2} \mathrm{~b}_{3}(P$. clavigera $75 \%$ ), tuvieron mejor efecto biocida con porcentajes de mortalidad de áfidos del $100 \%, 98 \%$ y 98 $\%$ respectivamente. Se determinó que para matar el $50 \%$ de los áfidos con extracto de $S$. mammosum se necesitó una dosis de $12.5 \%$ y el $\mathrm{DL}_{50}$ para el biocida $P$. clavigera fue $17.5 \%$ de concentración.

Palabras clave: Biocida, sacha yoco, teta de vaca, áfido, Solanum.

\section{ABSTRACT}

To determine the mean lethal dose $\left(\mathrm{LD}_{50}\right)$ under laboratory conditions of the extracts of Paullinia clavigera and Solanum mammosum in aphid mortality, treatments were applied: $T_{1}\left(a_{1} b_{1}=S\right.$. mammosum $\left.25 \%\right), T_{2}\left(a_{1} b_{2}=\right.$ S. mammosum $50 \%), \mathrm{T}_{3}\left(\mathrm{a}_{1} \mathrm{~b}_{3}=\mathrm{S}\right.$. mammosum $\left.75 \%\right), \mathrm{T}_{4}\left(\mathrm{a}_{1} \mathrm{~b}_{4}=\right.$ S. mammosum $\left.100 \%\right), \mathrm{T}_{5}\left(\mathrm{P}\right.$. clavigera $\mathrm{A}_{2} \mathrm{~B}_{1}$ $=25 \%), T_{6}\left(a_{2} b_{2}=P\right.$. clavigera $\left.50 \%\right), T_{7}\left(a_{2} b_{3}=P\right.$. clavigera $\left.75 \%\right), T_{8}\left(P\right.$. clavigera $\left.a_{2} b_{4}=100 \%\right)$ and $T_{9}$ (control); complete randomized design was used with factorial arrangement, of $2 \times 4+1$ additional control, with five replicates each treatment and determining the $\mathrm{DL}_{50}$, mortality results are consistent with the probit and cubic models. The parameters evaluated were: accumulative mortality of aphids, aphid population, number of alive for treatment and time the biocide application to the death of aphids. The doses factor had a significant influence on aphids mortality compared with the biocide factor was no significant. However, when factors interact mortality responses were different, so the treatments $\mathrm{a}_{1} \mathrm{~b}_{4}$ (S. mammosum $100 \%$ ), $\mathrm{a}_{2} \mathrm{~b}_{4}$ (S. clavigera $100 \%)$ and $\mathrm{a}_{2} \mathrm{~b}_{3}$ (P. clavigera $75 \%$ ), they had better biocida effect biocida with mortality rates aphids $100 \%, 98$ $\%$ y $98 \%$ respectively. It was determined to kill $50 \%$ aphids with the extract of S. mammosum, it was needed a dose of $12.5 \%$ while the $\mathrm{DL}_{50}$ for biocida P. clavigera was $17.5 \%$ of concentration.

Keywords: Biocide, soapberry, nipple fruit, aphid, cocona. 


\section{INTRODUCCIÓN}

Las plantas han evolucionado por más de 400 millones de años y para contrarrestar el ataque de los insectos han desarrollado mecanismos de protección como la repelencia y la acción insecticida (Cofre, 2011; Bonifaz,

2010). Diferentes mecanismos por los cuales, por un lado, atraen a insectos que son beneficiosos para ellas y por otro, se defienden frente al ataque producido por insectos fitófagos (García, 2004). Estos mecanismos de defensa que presentan las plantas pueden ser de tipo físico o químico, gracias a los cuales adquieren una serie de ventajas adaptativas (Boulter, 1993). Gran parte de las reacciones de defensa química se reflejan en una diversidad bioquímica que tiene muy pocos paralelos con otros grupos de organismos; la enorme diversidad fotoquímica y el largo tiempo de evolución de este metabolismo han resultado en interacciones de complejidad creciente. En el caso de las interacciones entre plantas e insectos, por ejemplo, ciertos compuestos con estructuras muy similares pueden ejercer actividades muy disimiles, desde insecticidas hasta repelentes e incluso atrayentes. Tamaña evolución de respuesta no solo resulta fascinante desde el punto de vista biológico, sino que también acarrea consecuencias económicas importantes (Vivanco et al., 2005).

Se han identificado más de 100000 metabolitos secundarios en plantas (Bhalla et al., 2005). Con la aparición en la década de los cuarenta de los insecticidas sintéticos, se pensó que los insecticidas vegetales desaparecerían para siempre pero el problema de la contaminación del ambiente, los residuos en el los alimentos y la resistencia por parte de los insectos han hecho que hoy en día vuelvan a ser tomados en cuenta (Bonifaz, 2010). Los fitoinsecticidas son una muy interesante alternativa de control de insectos, existen publicaciones que hacen un listado de plantas con propiedades insecticidas, documentando hasta 2500 especies de 247 familias botánicas (Silva et al., 2002).

La Amazonia peruana cuenta con una gran diversidad de especies vegetales, que, entre otros usos pueden ser utilizadas como biocidas. Estudios etnobotánicos en Ucayali, indican que el "sacha yoco" Paullinia clavigera var. bullata Simpson, Sapindaceae para ser investigados en el control de vectores primarios de la malaria, esencialmente (Anopheles benarrochi) y estudios sobre efecto insecticidas de "teta de vaca" Solanum mammosum, Solanaceae, en larvas de Prodiplosis longifila, en tomate; plantas seleccionadas como recursos botánicos con potencial biocida. Se cree que estas plantas biocidas podrían ejercer control sobre los áfidos que atacan al cultivo de cocona, debido a que tienen sustancias toxicas como alcaloides, esteroides, cardiotónicos, saponinas, taninos (Pérez 2004; Otoya, 2014) y triterpenos, flavonoides y quinonas (lannacone et al., 2000), con propiedades insecticidas que permite un buen control (Cañarte, 2000). El objetivo del presente trabajo de investigación fue determinar la dosis letal media $\left(D_{50}\right)$ en condiciones de laboratorio de los extractos vegetales preparados con "sacha yoco" Paullinia clavigera var. bullata Simpson y "teta de vaca" Solanum mammosum Dunal para control de áfidos que atacan al cultivo de Solanum sessiliflorum Dunal.

\section{MATERIALES y MÉTODOS}

\section{Crianza de afidios}

Se ubicaron plantas de Solanum sessiliflorum Dunal, en el campo con poblaciones de Aphis gossypii Glove; fueron colectados individuos adultos, libres de parásitos, de una parcela de cocona de ocho meses de edad, instalada en el Centro Experimental IIAP Huánuco, ubicada en la provincia de Leoncio Prado, Región Huánuco. Se escogieron ocho plantas libres de $A$. gossypii y de otros insectos, cuales se colocaron en una jaula cuadrada de $4 \mathrm{~m}^{2}$ con $1.70 \mathrm{~m}$ de altura, cubierta de malla antiafida con la finalidad de evitar el ingreso de otros insectos, a las cuales se las infectó con una población de 10 áfidos adultos. Después de 30 días fueron colectados 450 áfidos adultos en placas Petri y brotes de S. sessiliflorum, para la sobrevivencia de los individuos en laborator 


\section{Material vegetal}

Las hojas de $P$. clavigera fueron colectadas en Estación Experimental del Instituto de Investigaciones de la Amazonía Peruana (IIAP), distrito de Yarinacocha, provincia de Coronel Portillo, departamento de Ucayali, carretera Federico Basadre Km. 12.400. 8²4'18" S y 79³6'23" O. Los frutos de S. mammosum fueron colectados en Aucayacu, distrito de José Crespo y Castillo, provincia de Leoncio Prado, departamento de Huánuco, Latitud: -8.93139, Longitud: -76.1132.

\section{Extracto botánico}

Para la obtención del extracto madre de $S$. mammosum se maceró $100 \mathrm{~g}$ de frutos frescos en un litro de etanol al $96 \%$ durante siete días en un frasco de vidrio ámbar, periodo en el cual se agitó diariamente el frasco por diez segundos. El filtrado se realizó para separar el extracto obtenido de los residuos de materia seca. La dilución se realizó con agua destilada. El extracto de $P$. clavigera fue preparado con $100 \mathrm{~g}$ de biomasa (hoja molida) en una balanza y en un litro de agua destilada. Se picaron y trituraron las hojas; para realizar el macerado con un litro de agua hervida por un lapso 30 minutos, seguidamente se filtró en una tela fina, se descartó la torta. La relación cuantitativa $(\mathrm{p} / \mathrm{v})$ fue de 1:10 (100 $\mathrm{g}$ de biomasa seca por litro de solvente). Se realizó el proceso antes de la aplicación a los tratamientos. Al filtrado se le agregó agua destilada suficiente, de los cuales se obtuvieron los tratamientos a diferentes concentraciones (lannacone et al., 2008). Las dosis de aplicación se prepararon por dilución de una solución madre inicial de $100 \mathrm{ml}$ a la cual se le fue mezclando con agua para obtener la dosis experimental, es así que para obtener una dosis de $25 \%$ de cualquiera de los biocidas se tuvo que mezclar la solución madre con $75 \mathrm{ml}$ de agua, siguiendo este procedimiento las demás dosis (50\%, $75 \%$ y $100 \%$ ).

\section{Bioensayos}

Para determinar la actividad insecticida, se hizo aplicaciones de los biocidas a una dosis $25 \%$, $50 \%, 75 \%$ y $100 \%$, estas se efectuaron después de haber distribuido 10 pulgones en cada placa Petri, constituyendo cada placa una unidad experimental, se utilizó un frasco aspersor, donde se tuvo en cuenta la uniformidad en la aplicación y agitación constante del extracto. Las evaluaciones se realizaron a 1, 2, 3, 4, 5, 6, 7 y 8 horas después de la aplicación de los extractos. Para ello los resultados se expresaron en porcentajes de mortalidad acumulada, el cual nos sirvió para determinar la dosis letal media $\left(\mathrm{DL}_{50}\right)$ con los modelos probit y cúbico.

\section{Metodología estadística}

Los datos fueron analizados mediante el paquete estadístico Infostat. Se utilizó el DCA con arreglo factorial $2 \times 4+1$, con un testigo adicional, con cuatro tratamientos y seis repeticiones. Para determinar las comparaciones entre los tratamientos en estudio se utilizó la Prueba de Tukey $(\alpha=0.05)$ y para la aplicación de los modelos matemáticos se utilizó el software Microsoft Excel.

\section{RESULTADOS y DISCUSIÓN}

\section{Mortalidad de los áfidos}

Los resultados obtenidos de la prueba estadística ANOVA, para la mortandad de áfidos a cinco horas, después de la aplicación del extracto; el factor Biocida no presento influencia significativa en la mortalidad de los áfidos; sin embargo, el factor Dosis si mostró influencia significativa, como también su interacción de Biocida-Dosis, y Factores-testigo. Por tal motivo se realizó la prueba de comparaciones múltiples de Tukey, con un nivel de significancia de 0.05 , del cual se puede observar en la Tabla 1. Las dosis $75 \%$ y $100 \%$ no son significativamente diferentes, al igual que las dosis $50 \%$ y $25 \%$. 
Tabla 1. Prueba de comparaciones de Tukey para las dosis aplicadas de los extractos biocidas.

\begin{tabular}{cccc}
\hline \hline Dosis & Medias & & \\
\hline $100 \%$ & 99 & $\mathrm{a}$ & \\
$75 \%$ & 91 & $\mathrm{a}$ & \\
$50 \%$ & 82 & & $\mathrm{~b}$ \\
$25 \%$ & 77 & & $\mathrm{~b}$ \\
\hline \hline
\end{tabular}

Medias con una letra en común no son significativamente diferentes $(P>0.05)$

b: significancia $=0.371$

a: significancia $=0.057$

El análisis de comparación múltiple entre el factor Biocida - Dosis, presentado en la Tabla 2, muestra que los tratamientos T4, T8 y T7, no son significativamente diferentes entre sí; sin embargo, difieren estadísticamente con los tratamientos T3, T6, T2 y T5 que son significativamente similares entre sí. Estos resultados para el factor biocida indican que $P$. clavigera, con un porcentaje de mortalidad hasta un $98 \%$ a $75 \%$ y $100 \%$ de concentración del extracto vegetal puede tener efecto biocida. Celis et al. (2008), mencionan que los principales componentes que causan el efecto biocontrolador son metabolitos secundarios como terpenos, lignanos, alcaloides, esteroides, utilizado como mecanismo de defensa natural por la plantas frente al ataque microbiano o a la de predación de insectos o animales; Dixon (2001) y Ducrout (2005), indican que en Paullinia clavigera, presenta características fotoquímicas de los metabolitos secundarios con potencial biocida; al respecto lannacone et al. (2000), mencionan, que la efectividad de los insecticidas vegetales es dependiente de la sustancia orgánica empleada para su extracción y aplicación. Según lannacone y Pérez (2008), señalan que depende de los tipos de extractos; los extractos hexanicos obtenidos de $P$. clavigera son triterpenos, flavonoides, quinonas, de los extractos clorofórmicos se obtiene saponinas, esteroides, flavonoides, cumarinas y quinonas y de los extractos hidroalcoholicos saponinas, fenoles, flavonoides, cumarinas y quinonas; siendo los triterpenos responsables de la actividad tóxica. Cartaya y Reynaldo (2001) mencionan que algunos alcaloides son neurotóxicos a insectos y vertebrados herbívoros. Es así, como algunos metabolitos secundarios constituyen una parte importante de la respuesta de la defensa de las plantas sometidas al ataque por las plagas. Amadiola (2000), señala que las diferencias en la toxicidad de diferentes extractos pudieran deberse a la solubilidad de sus compuestos activos en los solventes o a la presencia de inhibidores activos en los solventes o de inhibidores de principios insecticidas. S. mammosum tiene un mayor porcentaje de mortalidad, se cree que es debido a su composición y modo de acción biocida ya que contiene taninos, saponoides, glicósidos cardiotónicos y saponinicos, esteroides, triterpenos y sesquiterpenlactona (Hernández et al., 1997); además, en el fruto contiene alcaloides esteroidales como solamargina y tomatina, asevarando una correlación de la toxicidad con el tipo de aglicona enlazado en la estructura alcaloidal (Alzerreca et al., 1981). S. mammosum, utilizado con disolventes como hexano, presentó diferencias de poca magnitud con el testigo para el control de Radopholus similis, baja mortalidad con el uso de metanol en intervalo de evaluación de 24, 48 y 72 horas; se consideró el disolvente con mayor potencial para compuesto nematicidas causando mortandad en todos los intervalos de evaluación de hasta un $76 \%$ y concluyendo que lo compuestos extraídos, fueron coumarinas, flavonoides y sesquiterpenlactonas con efecto biocidas, de S. mammosum (López, 2010). Resultados obtenidos en otros estudios indican la presencia de carbohidratos, compuestos fenólicos, esteroides y alcaloides (Barahona y Guevara, 2007, Pino, Sánchez y Rojas 2013); entre los tipos de alcaloides encontrados en esta especie están los alcaloides esteroidales (solasodina) y glucoalcaloide (Solanina). Estos alcaloides de naturaleza tretraciclica cuaternarios o terciarios son antifúngicos (Castillo et al., 2010); sin embargo, la solanina es un glucósido alcaloide muy tóxico que incluso en pequeñas cantidades, posee propiedades fúngicas y pesticidas, lo cual es una de las defensas naturales de la planta (Sepúlveda et al., 2003). 
Tabla 2. Comparación de medias (Duncan $\alpha=0.05$ ) del porcentaje acumulado de mortalidad de áfidos a las 5 horas después de la aplicación de los tratamientos.

\begin{tabular}{ccccccc}
\hline \hline Código & Clave & \multicolumn{2}{c}{ Tratamiento (aplicación) } & Mortalidad (\%) & \multicolumn{2}{c}{ Significancia } \\
\hline $\mathrm{T}_{4}$ & $\mathrm{a}_{1} \mathrm{~b}_{4}$ & Solanum mammosum & $100 \%$ & 100 & $\mathrm{a}$ & \\
$\mathrm{T}_{8}$ & $\mathrm{a}_{2} \mathrm{~b}_{4}$ & Paullinia clavigera & $100 \%$ & 98 & $\mathrm{a}$ & \\
$\mathrm{T}_{7}$ & $\mathrm{a}_{2} \mathrm{~b}_{3}$ & Paullinia clavigera & $75 \%$ & 98 & $\mathrm{a}$ & \\
$\mathrm{T}_{3}$ & $\mathrm{a}_{1} \mathrm{~b}_{3}$ & Solanum mammosum & $75 \%$ & 84 & & $\mathrm{~b}$ \\
$\mathrm{~T}_{6}$ & $\mathrm{a}_{2} \mathrm{~b}_{2}$ & Paullinia clavigera & $50 \%$ & 84 & & $\mathrm{~b}$ \\
$\mathrm{~T}_{2}$ & $\mathrm{a}_{1} \mathrm{~b}_{2}$ & Solanum mammosum & $50 \%$ & 80 & $\mathrm{~b}$ \\
$\mathrm{~T}_{1}$ & $\mathrm{a}_{1} \mathrm{~b}_{1}$ & Solanum mammosum & $25 \%$ & 80 & $\mathrm{~b}$ \\
$\mathrm{~T}_{5}$ & $\mathrm{a}_{2} \mathrm{~b}_{1}$ & Paullinia clavigera & $25 \%$ & 74 & $\mathrm{~b}$ \\
\hline \hline
\end{tabular}

\section{Evolución de la mortalidad de los áfidos}

La tendencia de la mortalidad obtenida con los extractos de Paullinia clavigera y Solanum mammosum a $100 \%, 75 \%, 50 \%$ y $25 \%$ se presenta en la Figura 1, respectivamente. La regresión con mejor ajuste en todos los casos fue polinomial de segundo grado, debido a que los biocidas tienen porcentajes de mortalidad crecientes formando una curva de mortalidad, ajustes polinómicos similares fueron obtenidos por Lizana (2005), cuando probó la eficiencia biocida de Melia azedarach L. sobre Anastrepha striata Schiner. En esta figura se observa que las mortalidades de los áfidos con los extractos en sus diferentes concentraciones son similares, así mismo se ve que algunos tratamientos llegan a producir la muerte de los áfidos gradualmente hasta las cinco horas, donde se presentó mayor mortalidad, después de la aplicación de los tratamientos, posteriormente hasta las ocho horas evaluada el testigo no presento mortalidad. Esta acción insecticida, se debería a una respuesta defensa de los biocidas, tanto espacial como temporalmente para una contención rápida del patógeno, conocida como respuesta sistémica (Sepúlveda et al., 2003). La mortalidad de áfidos con S. mammosum al $100 \%$ resulta mejor y evidente para todas las evaluaciones en comparación con las demás dosis de aplicación, mientras $P$. clavigera al $100 \%$ resulta tener el mismo comportamiento con $P$. clavigera al $75 \%$ al final de la evaluación. Por lo que se podría recomendar $P$. clavigera al $75 \%$ para el control de áfidos, puesto que tiene el mismo efecto, sin embargo, un análisis económico de los tratamientos en prueba sería más conveniente en la hora de realizar una recomendación. 

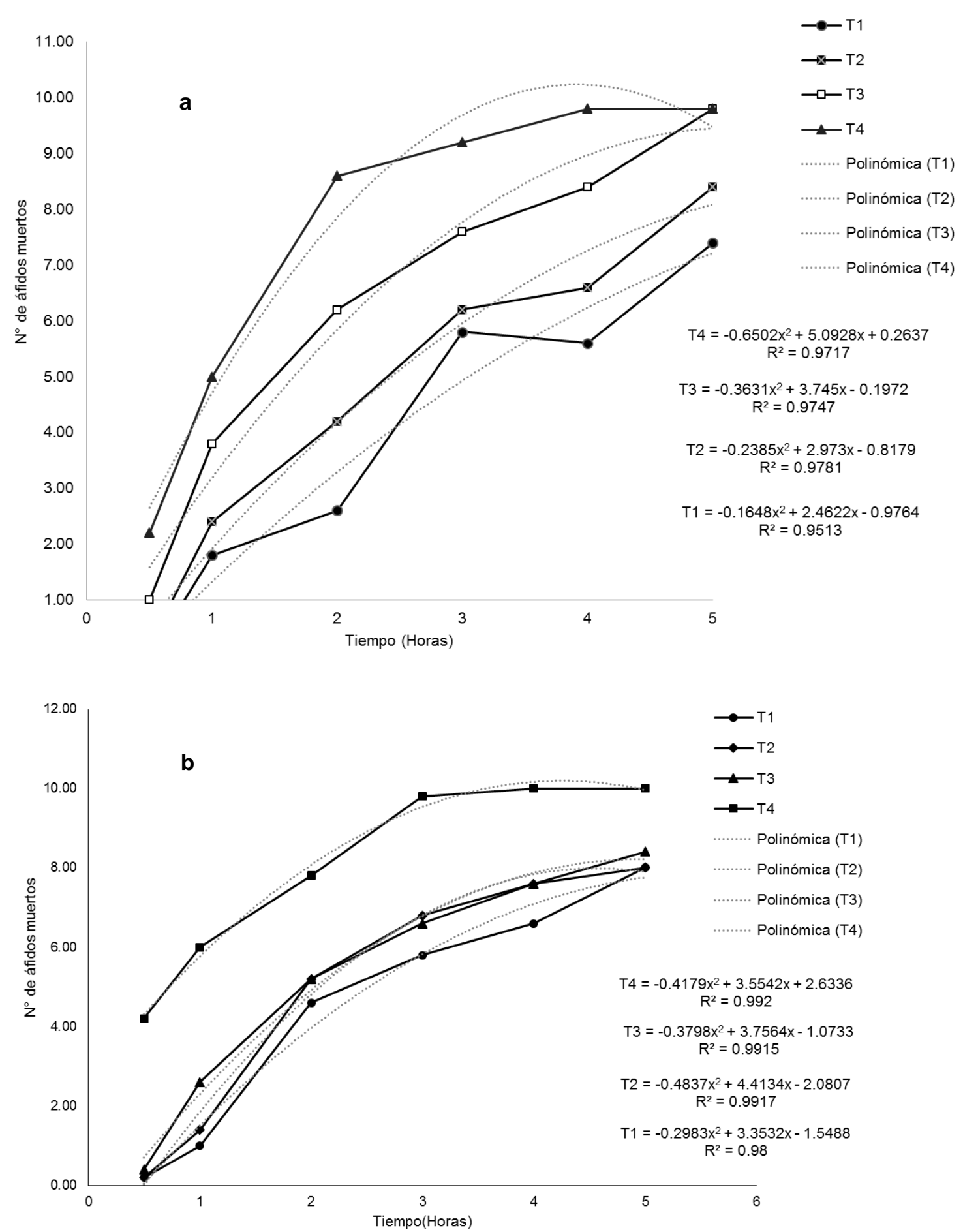

Figura 1. Evolución de la mortalidad de áfidos con extractos de Paullina clavigera (a) y Solanum mammosum (b) a diferentes concentraciones. 


\section{Dosis letal media}

Los resultados del ajuste al modelo probit y cúbico para determinar la $\mathrm{DL}_{50}$ del extracto de $S$. mammosum se presentan en la Figura 2 , se aprecia que el modelo cúbico $\left(R^{2}=0.99\right)$ se ajusta más a la curva de tendencia de mortalidad que el modelo probit $\left(R^{2}=0.95\right)$. Es decir, el modelo cúbico explica mejor la mortalidad de áfidos debido a que hay un buen grado de asociación entre los datos, por lo que la ecuación construida tiene una confiabilidad de del $99 \%$ para realizar estimaciones y predicciones este resultado es corroborado por Carmona-Hernández, et al (2014), quien menciona que los modelos no lineales explican mejor los fenómenos biológicos en comparación con los modelos lineales.
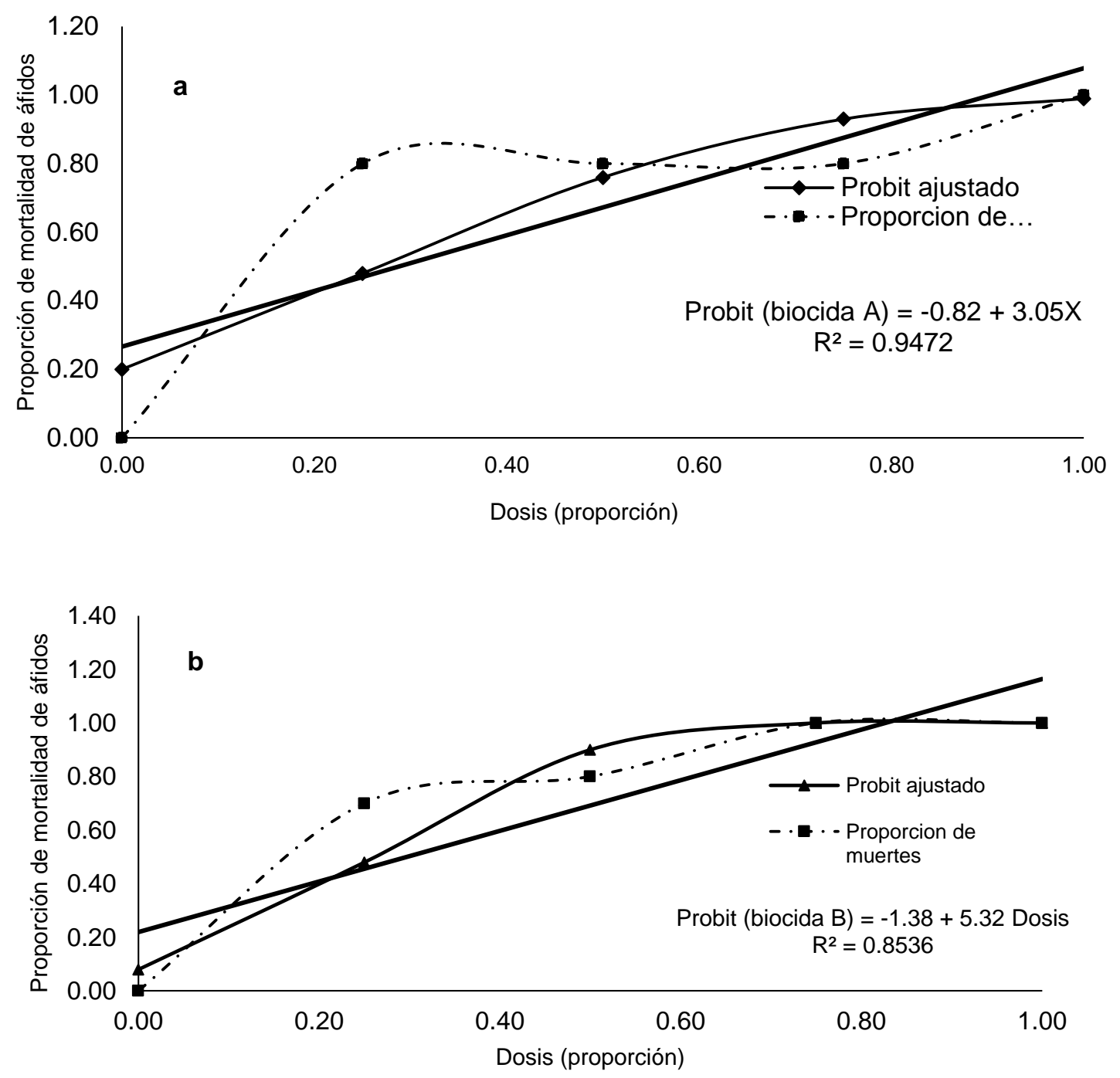

Figura 2. Proporción observada de muertes de áfidos según dosis crecientes de Solanum mammosum (a) y Paullinia clavigera (b) y modelo probit ajustado. 
Así mismo, se observa la $D L_{50}$ para el modelo probit y cúbico es de 27 y $12.5 \%$ respectivamente, lo que quiere decir que para matar el $50 \%$ de los individuos tratados en el experimento, se necesita aplicar extracto de S. mammosum al $27 \%$ cuando se utiliza el modelo probit y cuando se utiliza el modelo cúbico es tan solo de $12.5 \%$.

Si comparamos el efecto biocida de $P$. clavigera y $S$. mammosum utilizando el modelo probit basados en el DL50 resulta que no hay mucha diferencia significativa puesto que tienen $26 \%$ y $27 \%$ respectivamente, mientras si la comparación se hace utilizando el modelo cúbico hay diferencias significativas, debido a que para matar el $50 \%$ de los individuos de la muestra se necesita utilizar extracto de $P$. clavigera $(17.5 \%)$ a una concentración mayor que $S$. mammosum $(12.5 \%)$, estos resultados hacen que el modelo cúbico sea más confiable además tiene un coeficiente de correlación mayor que el modelo probit para ambos extractos. Sin embargo, si analizamos las respuestas (mortalidad) de la curva cúbica de la Figura 2a, indican que para matar $85 \%$ de áfidos se debe utilizar una dosis de $50 \%$ del extracto de S. mammosum, mientras que para matar un $77 \%$ de áfidos se necesita un $75 \%$ de concentración del extracto, es decir que a mayor dosis menor mortalidad de áfidos lo que sería incorrecto, por lo que el modelo se hace menos confiable al haber puntos de concavidad a lo largo de la curva. Este hecho es corroborado por Capra (2002), quien menciona que las ecuaciones no lineales sólo eran planteadas ocasionalmente, debido a que eran demasiado complejas y eran reemplazadas por aproximaciones lineales lo que ha conllevado a errores permanentes al creerse que los fenómenos naturales podrían ser descritos por ecuaciones lineales. Por otro lado, el modelo probit (Figuras $2 \mathrm{~b}$ y 3 ), sigue un patrón creciente, es decir que a mayor dosis hay mayor mortalidad áfidos debido a que no hay puntos de concavidad, dando como resultado coeficientes positivos, sin embargo el modelo no es confiable cuando se trata de estimar el DL50 pero se vuelve más confiable al incrementar la dosis de aplicación, coincidiendo con Casanoves et al. (2010), quienes mencionan que el análisis probit es el que más se ajusta para ensayos de plaguicidas, siempre que las dosis sean crecientes, por lo que el modelo en general es aceptable además los coeficientes de regresión son significativos.

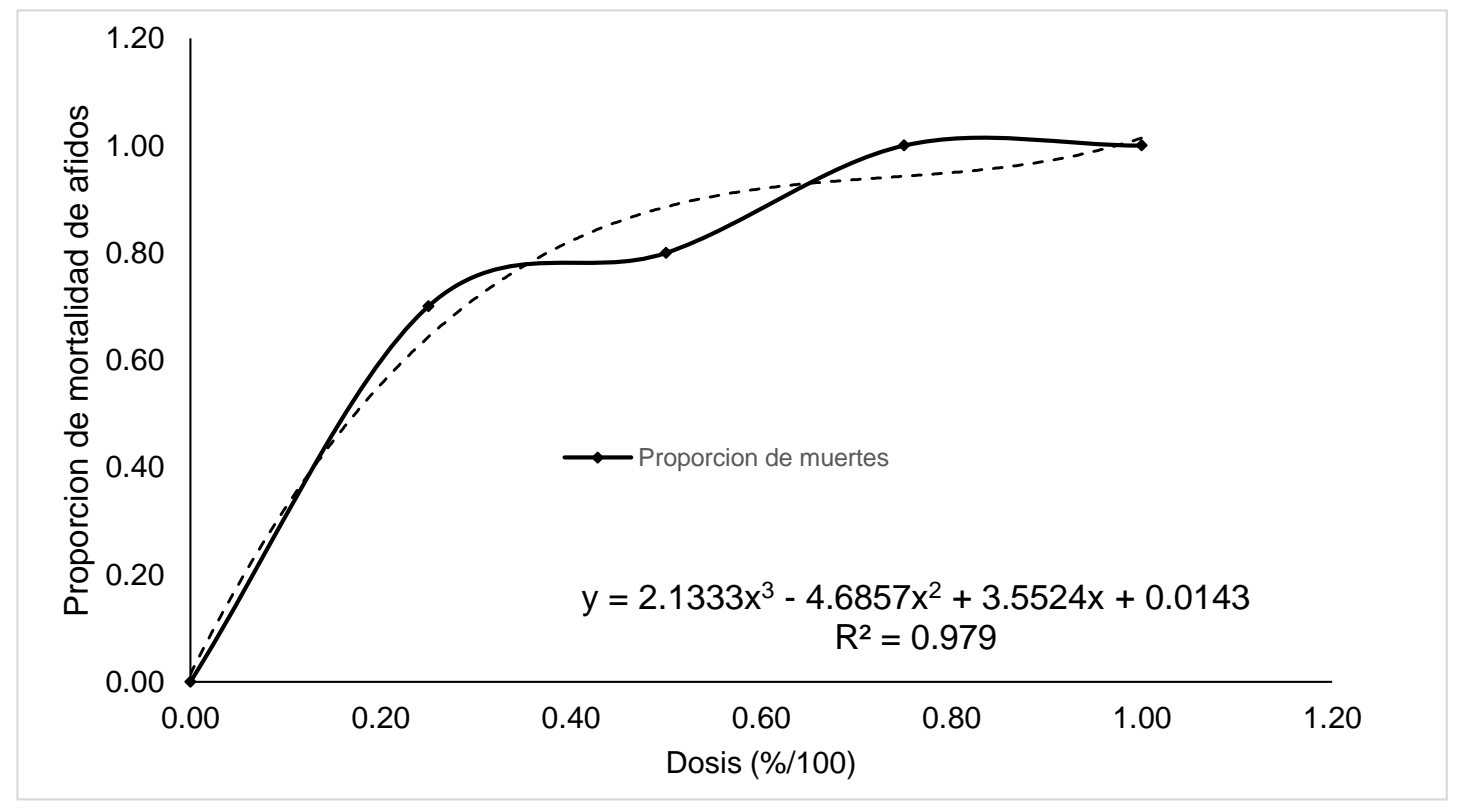

Figura 3. Proporción observada de muertes de áfidos según dosis crecientes de Paullinia clavigera y modelo cubico ajustado. 
Por todo lo mencionado, el modelo cúbico resulta mejor para calcular la dosis letal media, donde para matar el $50 \%$ de los áfidos con extracto de S. mammosum se necesita una dosis de 12.5 $\%$, mientras el DL50 para el biocida $P$. clavigera es $17.5 \%$ de concentración, resultando más tóxico el extracto de $S$. mammosum, este hecho coincide con Villalobos (1996) y Rodríguez (1997), quienes mencionan que mientras menor es la DL50, mayor es la toxicidad del producto.

\section{CONCLUSIONES}

Los tratamientos $\mathrm{a}_{1} \mathrm{~b}_{4}$ (Solanum mammosum $100 \%$ ), $\mathrm{a}_{2} \mathrm{~b}_{4}$ (Paullinia clavigera $\left.100 \%\right)$ y $\mathrm{a}_{2} \mathrm{~b}_{3}(P$. clavigera $75 \%$ ), tuvieron mejor efecto biocida con porcentajes de mortalidad de áfidos del 100 , 98 y $98 \%$ respectivamente, mientras que en los demás tratamientos los porcentajes de mortalidad fluctúan desde $84 \%$ en el tratamiento $a_{1} b_{3}$ (S. mammosum $75 \%$ ), hasta $74 \%$ en el tratamiento $\mathrm{a}_{2} \mathrm{~b}_{1}$ ( $P$. clavigera $25 \%$ ).

Para matar el 50 de los áfidos con extracto de S. mammosum se necesita una dosis de $12.5 \%$, mientras el DL50 para el biocida $P$. clavigera es $17.5 \%$ de concentración.

El factor dosis tuvo una influencia significativa en la mortalidad de áfidos en comparación con el factor biocida que resultó no significativo. Sin embargo, cuando interactúan los factores las respuestas a la mortalidad son diferentes.

\section{REFERENCIAS BIBLIOGRAFICAS}

1. Amadiola, A.C. 2000. Controlling rice blast in vitro and in vivo with extracts of Azadirachta indica. Crop Protección. 19: 452-460.

2. Alzérreca, A.; Arboleda, B.; Hart, G. 1981. Molluscicidal activity of natural products. The effect of Solanum glycosides alkaloids on Limnea cubensis Snails. J. Agric. Univ. P. R. 65:69-72.

3. Barahona, A., Guevara, B. 2007. Determinación de alcaloides esferoidales en extracto alcohólico el fruto de Solanum mammosum (chichigua) por cromatografía de capa fina. Tesis lic. El Salvador. Honduras, Universidad Tecnológica el Salvador. 90 pág.

4. Bhalla, R., Narasimham K., Swarup, S. 2005. Metabolomics and its role in understaning cellular responces in plants. Plant cell Rep 24: 562-571.

5. Bonifaz, P. 2010. Determinación de la actividad insecticida de la saponina de la quinua (Chenopodium quinoa) hidrolizada y no hidrolizada sobre (Drosophila melanogaster) en Riobamba. Tesis de Lic. Chimborazo, Ecuador. Escuela Superior Politécnica de Chimborazo. 69 p.

6. Boulter, D. 1993. Insect pest control by copying nature using genetically engineered crops. Phytochemistry: 34: 1453- 1456.

7. Castillo-Sanchez, L., Jiménezz-Osorni, J. y Delgado-Herrera, M. 2010. Secondary metabolites of the annonaceae, Solanaceae and Meliaceae families used as biological control of insects. Tropical and Subtropical Agroecosystems, 12(2010): 445 -462.

8. Casanoves, F.; Di Rienzo J.A.; Balzarini M.G.; González L.; Tablada M. y Robledo, C.W. 2010. InfoStat versión 2010. Grupo InfoStat, FCA, Universidad Nacional de Córdoba, Argentina. 200 p.

9. Capra, F. 2002. La trama de la vida. Una nueva perspectiva de los sistemas vivos. Anagrama. Barcelona, España. 65 p.

10. Cañarte, E. 2000. Oportunidad de los insecticidas vegetales en el manejo racional de cultivos rentables. Simposio Internacional de Manejo Racional de Insecticidas. Chillán, Chile. Pp. $24-47$. 
11. Carmona-Hernández, O., Fernandez, M. Palmeros -Sanchez, B., Lozada Garcia, J. 2014.Actividad insecticida de extractos etanolicos foliares de nueve piperaceas (Piper spp) en Drosophila melanogaster. Revista internacional de contaminación ambiental Vol. 30, 2014, pp 67-73.

12. Celis, A., Mendoza, C., Pachón, M., Cardona, J., Delgado, W. y Enrique, L. 2008. Extractos vegetales utilizados como biocontroladores con énfasis en la familia Piperaceae. Una revisión. Agronomía Colombiana. Volumen 26, Número 1, p. 97-106.

13. Cofre, S. 2011. Determinación de la actividad insecticida y/o antialimentaria del aceite esencial de Tsinzu (Tagetes minuta) en Drosophita melanogaster. Tesis, Lic. Riobamba, Ecuador. Escuela Superior Politécnica de Chimborazo. 88 p.

14. Cartaya, O., \& Reynaldo, I. 2001. Flavonoides: Características químicas y aplicaciones. Cultivos Tropicales, 22(2), 5-14.

15. Dixon, R. 2001. Natural products and plant visease resistance. Nature 411, $843-847$.

16. Ducrot, P.H. 2005. Organic chemistry's contributivos to the understanding of biopesticida activity of natural products from higher plants. pp. 47-58. En: Regnault, R.C., B.J.J. Philogeney C. Vincent (eds.). Biopesticides of plant origin. Lavoiser and Intercept, Ltd., Paris and Andover. $313 \mathrm{p}$.

17. Garcia, C. 2004. Efectos de terpenoides naturales y hemisinteticos sobre Leptinotarsa decenlineata (Say) (Coleoptera: Chrysomelidae) y Spodoptera exigua (Hubner) (Lepidoptera: Noctuidae). Memoria para optar el grado de doctor. Madrid. España. Universidad Complutense de Madrid. $119 \mathrm{p}$.

18. Hernández, A.; Sanabria, E.; Barrios, M. 1997. Extractos radicales de Bidens pilosa y Melampodium divaricatum y su efecto inhibitorio en la eclosión de huevos de Meloidogyne incognita. Annual Meeting APS-Caribbean Division, San José, CR. Universidad Nacional de Costa Rica. 25 p.

19. Iannacone, J., Alvariño, L. y Pérez, D. 2008. Efecto de Paullinia clavigera "Sacha yoco" (Sapindaceae) sobre la eclosión de huevos de Fasciola hepatica. Neotropical Helninthology, 2(2): $54-60$.

20. lannacone, J., y Pérez, D. 2008. Efecto toxico de cuatro plantas amazónicas sobre Chironomus calligraphus Goeldi 1905 (Diptera: Chironomidae) y Artemia franciscana. Kellog 1906 (Anostraca: Artemiidae). Revista Brasileira de Toxicología 21, n.1 (2008) 25 - 3.

21. Iannacone, J. y Lamas, G. 2000. Efecto insecticida de cuatro extractos botánicos y del cartap sobre la polilla de la papa Phthorimaea operculella (zeller) (Lepidoptera: Gelechiidae), en el Perú. Entomotropica. Vol. 18(2): 95-105. Agosto 2003.

22. Lizana, R.R. 2005. Elaboración y evaluación de extractos del fruto de Melia azedarach L. como insecticida natural. Tesis Ingeniero Forestal. Universidad de Chile. Santiago, Chile. 56 p.

23. López, A.2010. Evaluación de extractos de cuatro especies de plantas y sus compuestos orgánicos sobre la mortalidad de Radopholus similis en condiciones in vitro. Tesis. Lic. Costa Rica. Tecnológico de Costa Rica. 112 pag.

24. Otoya, L. 2014. Manejo de dos densidades de siembra y podas en la producción de Solanum mammosum L. (teta de vaca) y determinación de las propiedades insecticidas de los frutos. Tesis. Lic. Trujillo, Perú. Universidad Antenor Orrego. Trujillo, Perú. 86 p.

25. Pérez, D., lannacone, J. 2004. Efecto insecticida de sacha yoco (Paullinia clavigera var. bullata Simpson) (Sapindaceae) y oreja de tigre (Tradescantia zebrina Hort ex Bosse) (Commelinaceae) en el control de Anopheles benarrochi Gabaldon, Cova García y López, 1941, principal vector de malaria en Ucayali, Perú. FALTA LUGAR Y PAGINAS

26. Pino, O. Sánchez, Y. \& Rojas, M. 2013. Plant secondary metabolites as alternativas in pest management. II: An overview of their potential in Cuba. Revista de Protección Vegetal, 28(2), 95-108.

27. Rodríguez, C.; Silva, G. y Djair, V. 2003. Bases para el manejo racional de insecticidas: Insecticidas de origen vegetal. Facultad de Agronomía, Universidad de Concepción, y Fundación para la Innovación Agraria. Pp. 89 -111.

28. Rodríguez, H. 1997. Insecticidas y agricultura orgánica. Memoria del evento de aprobación en Certificación de Agricultura Orgánica, SAGAR-CP, Colegio de Postgraduados. Montecillo. Texcoco, México. Pp.162-197. 
29. Sepúlveda, J.G.; Porta, D.H. \& Rocha, S.M. 2003. La participación de los metabolitos secundarios en la defensa de las plantas. Revista Mexicana de Fitopatología. 21(3), 355363.

30. Silva, G.; Lagunes, A.; Rodríguez, J.C. y Rodríguez, D. 2002. Insecticidas vegetales: una vieja y nueva alternativa para el manejo de plagas. Manejo Integrado de Plagas y Agroecología (Costa Rica). 66: 4 - 12.

31. Vivanco, J., Cosio, E., Loyola, V., Flores, H. 2005. Mecanismos químicos de defensa de las plantas. Investigación y Ciencia. Barcelona, España. Vol. 341.68 p.

32. Villalobos, P. 1996. Plaguicidas naturales de origen vegetal: Estado actual de la investigación. Instituto Nacional de Investigación y Tecnología Agraria y Alimentaria, Madrid. $35 \mathrm{p}$. 